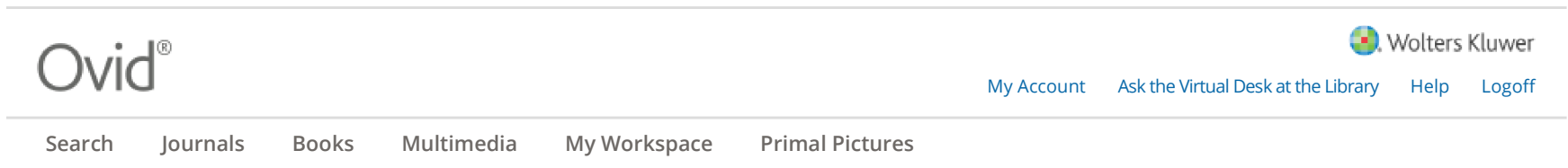

Search Results

\section{Critical Care Medicine}

Issue: Volume 24(2), February 1996, pp 274-279

Copyright: @ 1996 Wolters Kluwer Health I Lippincott Williams \& Wilkins

Publication Type: [Laboratory Investigation]

ISSN: 0090-3493

Accession: 00003246-199602000-00016

[Laboratory Investigation]

\section{Evaluation of lung function after intratracheal perfluorocarbon administration in healthy animals}

Tutuncu, Ahmet S. MD PhD; Houmes, Robert-Jan M. MD; Bos, Jelle A. H. MD PhD; Wollmer, Per MD PhD; Lachmann, Burkhard $M D P h D$

\section{Author Information}

From the Department of Anesthesiology (Drs. Tutuncu, Houmes, Bos, and Lachmann), Erasmus University Rotterdam, Rotterdam, The Netherlands; the Department of Anesthesiology (Dr. Tutuncu), Medical Faculty of University of Istanbul, Istanbul, Turkey; and the Department of Clinical Physiology (Dr. Wollmer), Malmo University Hospital, Lund University, Malmo, Sweden.

This study was performed at the Department of Anesthesiology, Erasmus University Rotterdam, The Netherlands, and supported, in part, by the International Foundation for Clinically Oriented Research, The Netherlands.

Address requests for reprints to: Dr. Burkhard Lachmann, Department of Anesthesiology (Room Ee 2393), Erasmus University Rotterdam, Postbox 1738, 3000 DR Rotterdam, The Netherlands.

\section{Abstract}

Objectives: To investigate the effects of partial liquid ventilation (i.e., mechanical ventilation in combination with intratracheal administration of perfluorocarbon) on lung function, with particular attention to the integrity of the alveolocapillary membrane in healthy adult animals.

Design: Prospective, randomized, controlled study.

Setting: Laboratory at the Department of Experimental Anesthesiology, Erasmus University Rotterdam.

Subjects: Ten adult male New Zealand rabbits.

Interventions: Five rabbits were intratracheally treated with $12 \mathrm{~mL} / \mathrm{kg}$ of perfluorocarbon while conventional mechanical ventilation (volume-controlled, tidal volume of $12 \mathrm{~mL} / \mathrm{kg}$, respiratory rate of $30 \mathrm{breaths} / \mathrm{min}$, inspiration/expiration ratio of $1: 2$, positive endexpiratory pressure of $2 \mathrm{~cm} \mathrm{H}_{2} \mathrm{O}$, and an $\mathrm{FIO}_{2}$ of 1.0) was applied for 3 hrs. To assess the permeability of the alveolocapillary membrane, pulmonary clearance of inhaled technetium-99m-labeled diethylenetriamine pentaacetic acid (sup 99m Tc-DTPA) measurements were performed at $3 \mathrm{hrs}$ and compared with data from the control group ( $\mathrm{n}$ equals 5 ) treated with mechanical ventilation only, using the same ventilatory parameters.

Measurements and Main Results: Pulmonary gas exchange and lung mechanical parameters were measured in both groups at 30 -min intervals. Mean values for $\mathrm{PaO}_{2}$ in the perfluorocarbon group, although at adequate levels, were less than those values of the control group during the 3 -hr study period ( 370 plus minus 44 vs. 503 plus minus 44 torr at 3 hrs [49.3 plus minus 5.9 vs. 67.1 plus minus $5.9 \mathrm{kPa}$ ]). Peak and mean airway pressures were higher in the perfluorocarbon group (ranging from 1.9 to $3.4 \mathrm{~cm} \mathrm{H}_{2} \mathrm{O}$ and 0.7 to $1.3 \mathrm{~cm} \mathrm{H}_{2} \mathrm{O}$, respectively) compared with the control group, while end-inspiratory airway pressure was similar in both groups. The half-

life of ${ }^{99 m}$ Tc-DTPA was 83.7 plus minus 24.5 mins in the control group, which was significantly longer ( $p$ less than .01) than in the perfluorocarbon group (49.8 plus minus 6.1 mins).

Conclusions: These findings suggest that partial liquid ventilation with perfluorocarbons lowers pulmonary gas exchange in healthy animals, and the increased pulmonary clearance of ${ }^{99 \mathrm{~m}}$ Tc-DTPA after 3 hrs of this type of ventilatory support may reflect minimal reversible changes in the lung surfactant system.

(Crit Care Med 1996; 24:274-279)

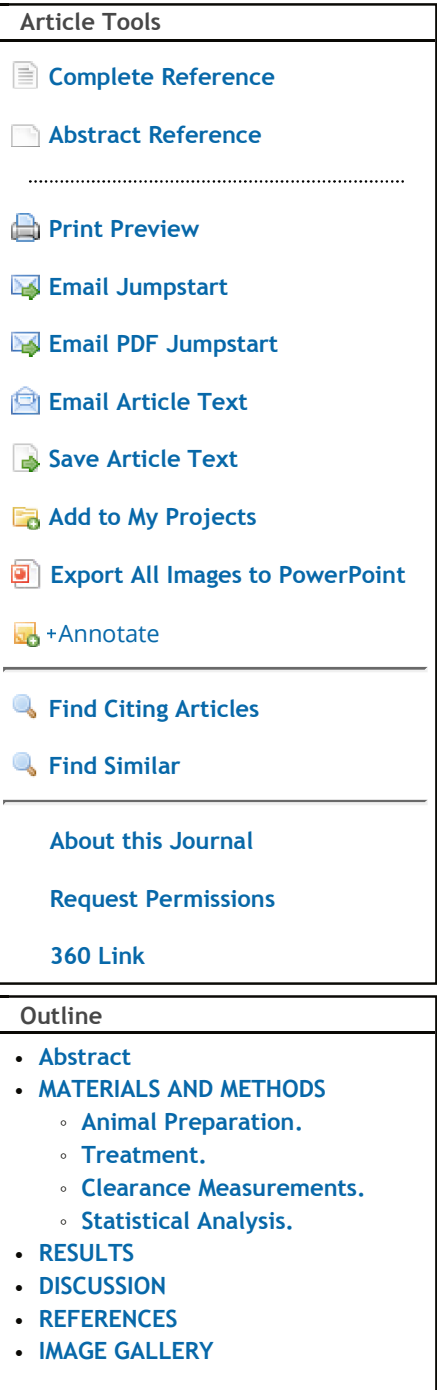


KEY WORDS: perfluorocarbon; Tc 99m-DTPA; pulmonary gas exchange; respiratory mechanics; mechanical ventilation; surface tension; pulmonary surfactants; lungs; pulmonary emergencies

Studies [1-5] with perfluorocarbon total liquid ventilation have shown this technique to be an effective means of respiratory support to provide effective pulmonary gas exchange and to improve lung function in animals with acute respiratory failure. As an alternative perfluorocarbon application method to those methods requiring a liquid ventilation system, as referenced in these studies [1-5], we [6] demonstrated that intratracheal perfluorocarbon administration in combination with conventional mechanical ventilation (partial liquid ventilation) improves pulmonary gas exchange in a dose-related manner in an animal model of acute respiratory failure. Other studies [7], using the same approach, indicated that effective respiratory support can be maintained for several hours at high perfluorocarbon doses. Moreover, lung structure is well maintained in animals with acute respiratory failure [8].

The effects of total liquid ventilation on healthy lungs, pulmonary gas exchange, lung mechanics, and cardiovascular dynamics have been investigated in healthy animals. Arterial oxygenation was consistently lower in healthy animals during total liquid ventilation, although these oxygenation values were at satisfactory levels. At present, partial liquid ventilation in healthy lungs has been attempted in only a few animal experiments. Using mechanical ventilation in combination with intratracheal administration of perfluorocarbon, Fuhrman et al. [9] demonstrated that after filling the lungs with perfluorocarbon to a volume of functional residual capacity, adequate pulmonary gas exchange can be provided in healthy animals.

Since this new technique differs from total liquid ventilation and employs conditions applied to both liquid and gas physics, this technique of partial liquid ventilation needs to be further investigated with more sensitive and detailed methods, especially with respect to its safety for normal lung function and also compared with other available ventilatory support techniques.

Because integrity of the alveolocapillary barrier is an important factor for normal function of the respiratory system, there is great interest in measuring any changes to this barrier function, e.g., by measuring the pulmonary clearance of radiolabeled hydrophilic solutes. Measurement of the pulmonary clearance of technetium-99m-labeled diethylenetriamine pentaacetic acid (sup 99m Tc-DTPA) is proven to be a sensitive, fast, and noninvasive technique to detect the integrity of the alveolocapillary barrier $[10,11]$. Moreover, the clearance rate of ${ }^{99 m}$ Tc-DTPA mainly depends on the functional integrity of the surfactant system $[12,13]$. Thus, in order to evaluate the effects of partial liquid ventilation on lung function (gas exchange and lung mechanics), and specifically on the integrity of the alveolocapillary barrier, we tested the clearance of ${ }^{99 \mathrm{~m}}$ Tc-DTPA from the lungs in healthy animals after $3 \mathrm{hrs}$ of partial liquid ventilation and compared these data with data from animals receiving conventional gas ventilation.

Back to Top

\section{MATERIALS AND METHODS}

This study was approved by the Animal Committee of Erasmus University Rotterdam

Back to Top

Animal Preparation.

Ten adult New Zealand rabbits (mean body weight of 2.2 plus minus $0.2 \mathrm{~kg}$ ) were anesthetized with an injection of pentobarbital sodium $(50 \mathrm{mg} / \mathrm{kg}$ iv) via an auricular vein. The animals were positioned supine and a tracheostomy was performed. Volume-controlled ventilation (Servo 900C, Siemens-Elema, Solna, Sweden) was initiated with a tidal volume of $12 \mathrm{~mL} / \mathrm{kg}$, respiratory rate of 30 breaths/min, inspiration/expiration ratio of $1: 2$, positive end-expiratory pressure (PEEP) of $2 \mathrm{~cm} \mathrm{H} \mathrm{H}_{2} \mathrm{O}$, and an FIO sub 2 of 1.0 . Anesthesia was maintained with additional doses of pentobarbital, as required. Pancuronium bromide was administered by continuous infusion $(0.1 \mathrm{mg} / \mathrm{kg} / \mathrm{hr})$ for muscle paralysis. A maintenance fluid of $5 \%$ dextrose and $0.45 \%$ sodium chloride was administered 7.5 $\mathrm{mL} / \mathrm{kg} / \mathrm{hr}$ ). A carotid artery was cannulated for blood pressure monitoring and blood sampling.

After animal preparation and baseline measurements were performed, the animals were randomized into two groups of five animals each. In one group, a volume of $12 \mathrm{~mL} / \mathrm{kg}$ of perfluorocarbon was administered intratracheally in two 
subsequent volumes as a bolus (at 3-min intervals). At the time of perfluorocarbon instillation, the animals were disconnected from the ventilator. The animals were reconnected immediately after perfluorocarbon administration and volumecontrolled ventilation was continued. The second group received no treatment but was mechanically ventilated only. The study protocol was limited to $3 \mathrm{hrs}$ because the animals were observed to be free of visible perfluorocarbon at the endotracheal tube during disconnection from the ventilator at $3 \mathrm{hrs}$ (unpublished observations). Both groups received volume-controlled ventilation for $3 \mathrm{hrs}$, using the same ventilator settings as described above: FIO sub 2 of 1.0 ; tidal volume of $12 \mathrm{~mL} / \mathrm{kg}$; respiratory rate of 30 breaths/min; inspiration/expiration ratio of 1:2; and PEEP of 2 $\mathrm{cm} \mathrm{H}_{2} \mathrm{O}$.

During the study period, blood gases and $\mathrm{pH}$ were measured (ABL-330, Radiometer, Copenhagen, Denmark), and respiratory mechanics (airway pressures, airway resistances) were recorded by a lung mechanics calculator ( 940 , SiemensElema) [14] at 15- and 30-min intervals thereafter. At the end of the 3-hr observation period, clearance measurements of ${ }^{99 m}$ Tc-DTPA were performed.

Back to Top

Treatment.

All studies were performed, using perflubron (Liquivent Trademark [perfluorooctyl bromide], Alliance Pharmaceutical Corporation, San Diego, CA).

Perflubron is a perfluorocarbon with a specific gravity of $1.918 \mathrm{~g} / \mathrm{cm}^{3}$ at 25 degrees $\mathrm{C}$, surface tension of $18.1 \mathrm{dyne} / \mathrm{cm}$, vapor pressure of 3.6 torr $(0.5 \mathrm{kPa})$ at 20 degrees $C$ and 10.5 torr $(1.4 \mathrm{kPa})$ at 37 degrees $C$, oxygen solubility of $53 \mathrm{~mL} / 100$ $\mathrm{mL}$, and $\mathrm{CO}_{2}$ solubility of $210 \mathrm{~mL} / 100 \mathrm{~mL}$ at 37 degrees $\mathrm{C}$ and 1 atmosphere of pressure.

Back to Top Clearance Measurements.

The animals were placed under a gamma camera and were kept under the same anesthesia protocol as described above. Radioactivity was measured in the anterior view.

A solution of ${ }^{99 m}$ Tc-DTPA, propared from a commercial kit (Technescan DTPA, Mallinckrodt Medical, Petten, The Netherlands), was nebulized into the inspiratory line of the ventilation circuit, as described by Dahlback et al. [15], using an air jet nebulizer (Ultravent Trademark, Mallinckrodt Medical). This type of nebulizer is designed to produce aerosols with droplets in the range of 0.6 to 2 micro meter, favoring alveolar deposition [16]. The supply of pressurized air to the nebulizer was controlled by a pneumatic valve that was connected to the ventilator along with an electronic circuit. The nebulizer operated only during expiration, filling the inspiratory line with aerosol in order to administer the particles with the ensuing insufflation. Before running the nebulizer, the system was checked for airleaks in each animal. During aerosol administration, all animals in both groups received pressure-controlled ventilation with a peak inspiratory pressure equal to the final recording of the end-inspiratory airway pressure during volume-controlled ventilation, and a respiratory rate of 30 breaths/min, inspiration/expiration ratio of $1: 1$, and zero end-expiratory pressure. These ventilatory settings can create an intrinsic PEEP of $2 \mathrm{~cm} \mathrm{H}_{2} \mathrm{O}$ (identical to the experimental set-PEEP level). High radioactivity over the lungs can be achieved within 1 to 2 mins [17]. When a count rate of approximate 300 to 400 counts/sec over the lungs was obtained, aerosol administration was discontinued, the experimental ventilation settings (volumecontrolled) were resumed, and clearance measurements were started immediately. Gamma camera images were obtained in successive 1-min frames for 20 mins and stored in a computer (PDP 11/34, Digital, Maynard, MD).

Data from the clearance measurements from both lungs of each animal were analyzed and a time-activity curve was generated. A monoexponential function was fitted to the experimental data and the half-life of the tracer in the lungs was calculated. After completion of the measurements, the animals were killed with an overdose of pentobarbital.

Back to Top

Statistical Analysis.

All data are expressed as mean plus minus SD. The repeated measurements were compared by multifactor analysis of variance, using the multiple range test. Data for nonrepetitive measurements were analyzed by Student's t-test. A p less than .05 indicated a statistically significant difference. 


\section{RESULTS}

All animals survived the study period. The baseline values for the measured variables were comparable in both groups.

During the 3 hrs of mechanical ventilation with intratracheal perfluorocarbon administration, $\mathrm{PaO}_{2}$ values decreased (27\%) compared with baseline values and the mean data for $\mathrm{PaO}_{2}$ were significantly lower compared with the control group throughout the study period Table $1 . \mathrm{PaCO}_{2}$ and arterial $\mathrm{pH}$ data were comparable in both groups, with slightly higher $\mathrm{PaCO}_{2}$ and lower arterial $\mathrm{pH}$ values in the perfluorocarbon group Table 1 . The mean data for arterial pressures and heart rate remained unchanged in both groups.

< class="fulltext-GRAPHIC">TT1_95_42_0_Table $1<$ LINK GX Table 1</LINK>. Arterial blood gases, $\mathrm{pH}$, and hemodynamic data of the perfluorocarbon $(\mathrm{P})$ and control $(\mathrm{C})$ groups (mean plus minus SD)

Data for respiratory system mechanics are shown in Table 2. Peak airway pressure increased after perfluorocarbon administration and led to a slight increase (range 0.7 to $1.3 \mathrm{~cm} \mathrm{H}_{2} \mathrm{O}$ ) in mean airway pressure compared with the control group. The endinspiratory pause airway pressure was comparable in both groups during the study period. The inspiratory airway resistance in the perfluorocarbon group was significantly higher compared with the control group, whereas expiratory airway resistance values did not differ between the two groups.

< class="fulltext-GRAPHIC">TT2_96_44_0_Table $2<$ LINK GX Table 2</LINK>. Respiratory system mechanics of the perfluorocarbon $(\mathrm{P})$ and control $(\mathrm{C})$ groups (mean plus minus SD)

Distribution of the nebulized ${ }^{99 m}$ TC-DTPA aerosol in the lungs was uniform on analog images in all animals. The clearance curves of ${ }^{99 m}$ TC-DTPA from the two groups are shown in Figure 1. The faster pulmonary clearance of the tracer is seen in the time-activity curve of the perfluorocarbon group. The mean half-life of the tracer in the control group was 83.7 plus minus 24.5 mins (range 54.9 to 125.3), which was significantly greater than the mean half-life in the perfluorocarbon group (49.8 plus minus 6.1 mins [range 42.9 to 59.5 ])

< class="fulltext-GRAPHIC">FF1_97_46_0_Figure $1<$ LINK GX Figure 1</LINK>. Timeactivity curves of technetium-99m-labeled diethylenetriamine pentaacetic acid in the perfluorocarbontreated (inverted triangles) and the control (squares) groups. Values are expressed as mean plus minus SD.

Back to Top

\section{DISCUSSION}

This study provides the first experimental data on assessment of alveolocapillary barrier function by means of pulmonary clearance measurements with an aerosol radiolabeled tracer after administering a perfluorocarbon liquid into healthy animal lungs, and demonstrates an increased clearance rate of ${ }^{99 m}$ Tc-DTPA after $3 \mathrm{hrs}$ of partial liquid ventilation compared with mechanical ventilation only.

The study demonstrates that mechanical ventilation in combination with perfluorocarbon alters pulmonary gas exchange in healthy animals. Our observation that arterial oxygenation is decreased during partial liquid ventilation is consistent with that finding in the first report [9], using this new ventilatory support technique in healthy animals, as documented by Fuhrman et al. Fuhrman's group demonstrated similar decreases in arterial oxygenation when compared with conventional gas ventilation, although adequate oxygen levels were maintained in that study as well.

In healthy lungs, the alveolocapillary barrier (which consists of alveolar surfactant layer, alveolar epithelium, basement membrane, and capillary endothelium) protects the transfer of solutes and proteins across the barrier. However, when the integrity of this barrier is altered, permeability is increased, allowing high permeability edema to develop [18]. Previous studies $[19,20]$ indicated that the integrity of the alveolocapillary membrane is disturbed in patients with adult or infant respiratory distress syndrome, and clearance rates of the radiotracers correlate well with the stage (improvement) of the lung pathology. The clearance rate of ${ }^{99 m}$ Tc-DTPA has been reported to be changed by various factors, such as in response to altered surface tension in the lungs [21,22], and in response to increases in lung volume (i.e., high PEEP or large tidal volume ventilation) [23-25].

On intratracheal administration, perfluorocarbon distributes in the lung by acting on the alveolar air-liquid interface and by filling the alveoli, depending on the volume administered [6-8]. After administration of perfluorocarbon (possessing a constant surface tension), the surface tension characteristics of surfactant at the 
alveolar air-liquid interface are altered (or governed by surface tension from perfluorocarbon) and a new film with a new surface tension is established at the alveolar air-liquid interface due to evaporation of the perfluorocarbon (presumably higher than alveolar surfactant at end-expiration). This mechanism of action has been indirectly supported by a study conducted on healthy animals, in which arterial oxygenation remained unchanged when higher PEEP levels were applied, using the same experimental ventilator settings (unpublished observations). Changes in the surface tension properties of the lung may be associated with changes in the permeability of alveolar epithelium $[22,26]$. Thus, the increased surface tension at the alveolar-air interface in the expiratory phase in perfluorocarbon-treated animals might have contributed to the increased clearance rate of ${ }^{99 m}$ Tc-DTPA in the healthy lungs.

The clearance of ${ }^{99 m}$ TC-DTPA is a sensitive indicator of alterations in the pulmonary surfactant system [13,17,21]. Evander and co-workers [21] demonstrated that a detergent (dioctyl sodium sulfosuccinate), which acts only on the surfactant system and causes no structural damage to the alveolar epithelium [27], increases the clearance rate of ${ }^{99 m}$ Tc-DTPA without any observed changes in blood gases and lung mechanics. Bos et al. [13] demonstrated that experimentally increased alveolar surfactant content reduces the clearance rate of ${ }^{99 m}$ Tc-DTPA in healthy animals, indicating the role for the surfactant system in limiting the alveolocapillary transfer of solutes. The high sensitivity of clearance measurements of ${ }^{99 m}$ Tc-DTPA is an important point for consideration during interpretation of the present results. In one study [13], Bos and colleagues showed an increased clearance rate of ${ }^{99 m}$ Tc-DTPA in healthy animals after introducing into the lungs a volume of saline as small as $5 \mathrm{~mL}$. They [13] suggested that this increased clearance rate was due to changes in lung surfactant function. Therefore, one has to consider the possible interaction between the perfluorocarbon liquid and the lung surfactant in healthy animals. On the other hand, the hydrophobic and lipophobic perfluorocarbon liquid might impose another diffusion barrier to the hydrophilic tracer agent, ${ }^{99 m}$ Tc-DTPA, which has an extremely low lipid solubility. However, if this situation was the case, we could measure a slower clearance rate in the perfluorocarbon group compared with the control group, in contradiction to the results of the present study.

The effects of increased lung volume on lung permeability in healthy animals have been studied during conventional gas ventilation. The clearance rate of ${ }^{99 m} \mathrm{Tc}$ DTPA remained at the baseline value at a PEEP of $2.5 \mathrm{~cm} \mathrm{H}_{2} \mathrm{O}$, while increased PEEP levels of more than $5 \mathrm{~cm} \mathrm{H}_{2} \mathrm{O}$ increased the clearance rate in healthy animals [23]. In another study [28] in healthy animals, moderate PEEP values (2 to $10 \mathrm{~cm} \mathrm{H}_{2}$ O) had little effect on the clearance rate as compared with marked increases at a PEEP of $15 \mathrm{~cm} \mathrm{H}_{2} \mathrm{O}$. Furthermore, high lung volumes--not airway pressure--increased the clearance rate of the tracer element [29]. In the present study, we applied the least possible value of PEEP $(2 \mathrm{~cm} \mathrm{H}$ O) to prevent both the bulk movement of perfluorocarbon liquid along the airways and to eliminate the possible increases in lung volume that might arise from high PEEP values. On the other hand, although the ventilation volumes were equal, there were only trivial differences in the insufflation pressures between the groups in this study. The increased peak airway pressure in the perfluorocarbon group was probably a reflection of high airway resistance during inspiration, since airway resistance was increased in this group after perfluorocarbon administration. Moreover, the end-inspiratory airway pressures, which might be taken as a reflection of transthoracic pressure, did not significantly differ between the two groups during the study period. One might argue that the presence of a dense liquid (such as perfluorocarbon) in the lungs might act as an additional PEEP, causing further lung distention. Although there were no exact data on lung volume in the perfluorocarbon group, the mean airway pressure, which may be taken as an estimate of lung expansion (volume), was slightly higher in the perfluorocarbon group compared with the control group. Based on the above discussion, whether this slight difference in pressure of approximate 1 $\mathrm{cm} \mathrm{H}_{2} \mathrm{O}$ between the mean airway pressures may have contributed to the increased clearance rate of the tracer remains to be further evaluated.

Regarding the above-mentioned discussion and based on our present experimental findings, we speculate that the increased permeability for small molecules in healthy animals may be due to the alterations in the surfactant system function after application of perfluorocarbon liquids. However, healthy animals treated with the same protocol could be restored to spontaneous breathing (after 3 hrs of partial liquid ventilation) without any changes in lung function by day 7 after the study (unpublished observations). Furthermore, lung tissue from histologic studies in healthy animals appeared to be normal within a few days after liquid ventilation [30], and remained normal even after 3 yrs [31,32]. Considering these points, it is likely that healthy animal lungs are capable of reversing any changes in 


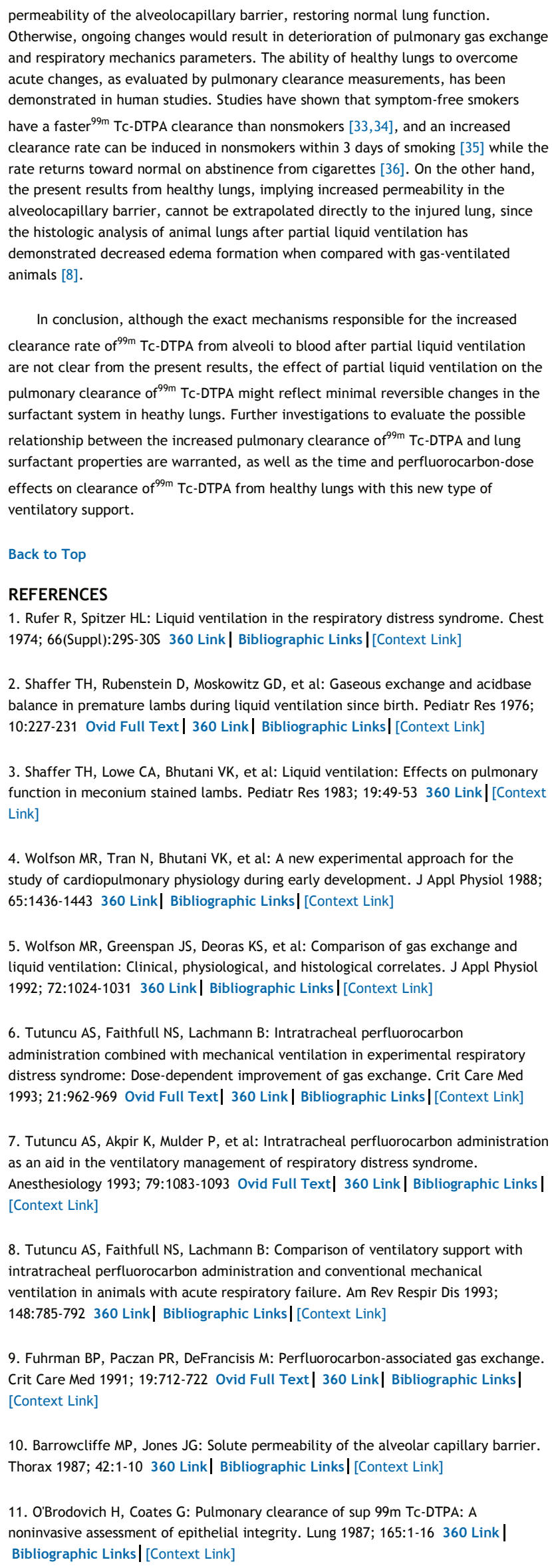

1. Rufer R, Spitzer HL: Liquid ventilation in the respiratory distress syndrome. Chest 1974; 66(Suppl):29S-30S 360 Link| Bibliographic Links|[Context Link]

2. Shaffer TH, Rubenstein D, Moskowitz GD, et al: Gaseous exchange and acidbase balance in premature lambs during liquid ventilation since birth. Pediatr Res 1976; 10:227-231 Ovid Full Text | 360 Link| Bibliographic Links| [Context Link]

3. Shaffer TH, Lowe CA, Bhutani VK, et al: Liquid ventilation: Effects on pulmonary function in meconium stained lambs. Pediatr Res 1983; 19:49-53 360 Link|[Context Link]

4. Wolfson MR, Tran N, Bhutani VK, et al: A new experimental approach for the study of cardiopulmonary physiology during early development. J Appl Physiol 1988; 65:1436-1443 360 Link| Bibliographic Links| [Context Link]

5. Wolfson MR, Greenspan JS, Deoras KS, et al: Comparison of gas exchange and liquid ventilation: Clinical, physiological, and histological correlates. J Appl Physiol 1992; 72:1024-1031 360 Link| Bibliographic Links|[Context Link]

6. Tutuncu AS, Faithfull NS, Lachmann B: Intratracheal perfluorocarbon administration combined with mechanical ventilation in experimental respiratory distress syndrome: Dose-dependent improvement of gas exchange. Crit Care Med 1993; 21:962-969 Ovid Full Text| 360 Link| Bibliographic Links|[Context Link]

7. Tutuncu AS, Akpir K, Mulder P, et al: Intratracheal perfluorocarbon administration as an aid in the ventilatory management of respiratory distress syndrome. Anesthesiology 1993; 79:1083-1093 Ovid Full Text| 360 Link| Bibliographic Links| [Context Link]

8. Tutuncu AS, Faithfull NS, Lachmann B: Comparison of ventilatory support with intratracheal perfluorocarbon administration and conventional mechanical ventilation in animals with acute respiratory failure. Am Rev Respir Dis 1993; 148:785-792 360 Link| Bibliographic Links| [Context Link]

9. Fuhrman BP, Paczan PR, DeFrancisis M: Perfluorocarbon-associated gas exchange. Crit Care Med 1991; 19:712-722 Ovid Full Text| 360 Link| Bibliographic Links| [Context Link]

10. Barrowcliffe MP, Jones JG: Solute permeability of the alveolar capillary barrier. Thorax 1987; 42:1-10 360 Link| Bibliographic Links| [Context Link]

11. O'Brodovich H, Coates G: Pulmonary clearance of sup 99m Tc-DTPA: A noninvasive assessment of epithelial integrity. Lung 1987; 165:1-16 360 Link | Bibliographic Links | [Context Link] 


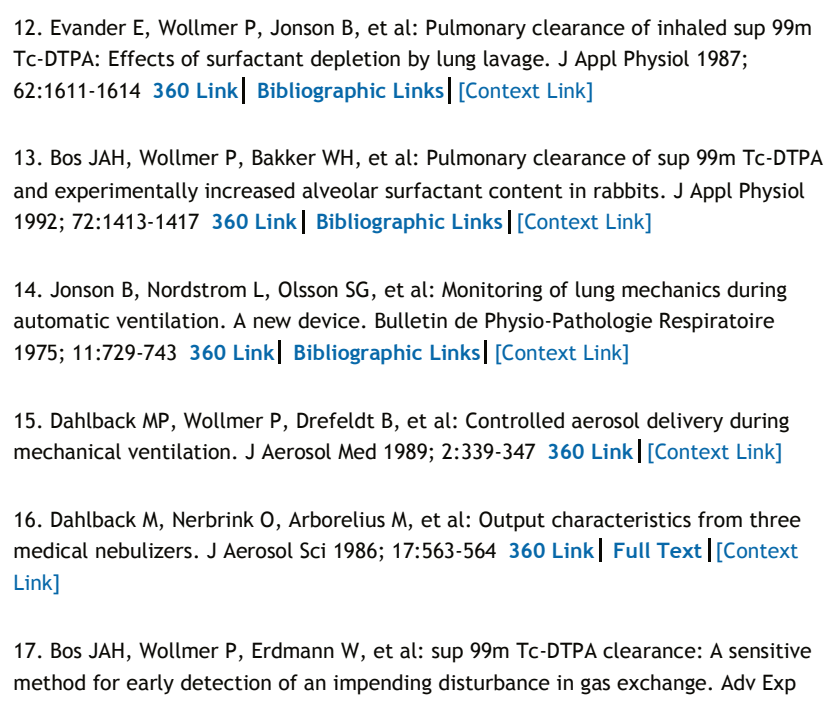

19. Mason GR, Effros RM, Uszler JM, et al: Small solute clearance from the lungs of patients with cardiogenic and noncardiogenic pulmonary edema. Chest 1985; 88:327-334 360 Link| Bibliographic Links| [Context Link]

20. Jefferies AL, Coates G, O'Brodovich H: Pulmonary epithelial permeability in hyaline-membrane disease. N Engl J Med 1984; 311:1075-1080 360 Link| Bibliographic Links | [Context Link]

21. Evander $E$, Wollmer $P$, Jonson B: Pulmonary clearance of inhaled sup $99 \mathrm{~m}$ Tc DTPA: Effect of the detergent dioctyl sodium sulfosuccinate in aerosol. Clin Physiol 1988; 8:105-111 360 Link| Bibliographic Links|[Context Link]

22. Jefferies AL, Kawano T, Mori S, et al: Effect of increased surface tension and assisted ventilation on sup 99m Tc-DTPA clearance. J Appl Physiol 1988; 64:562-568 360 Link| Bibliographic Links| [Context Link]

23. Cooper JA, van der Zee H, Line BR, et al: Relationship of end-expiratory pressure, lung volume, and sup 99m TC-DTPA clearance. J Appl Physiol 1987; 63:1586-1590 360 Link| Bibliographic Links| [Context Link]

24. Borrowcliffe MP, Zanelli GD, Jones JG: Pulmonary clearance of radiotracers after positive end-expiratory pressure or acute lung injury. J Appl Physiol 1989; 66:288294 [Context Link]

25. Marks JD, Luce JM, Lazar NM, et al: Effect of increases in lung volume on clearance of aerosolized solute from human lungs. J Appl Physiol 1985; 59:1242-1248 360 Link| Bibliographic Links| [Context Link]

26. Guyton AC, Moffatt DS, Adair TH: Role of alveolar surface tension in transepithelial movement of fluid. In: Pulmonary Surfactant. Robertson B, van Golde LMG, Batenburg JJ (Eds). Amsterdam, Elsevier, 1984, pp 171-185 [Context Link]

27. Nieman GF, Bredenberg CE: High surface tension pulmonary edema induced by detergent aerosol. J Appl Physiol 1985; 58:129-136 [Context Link]

28. O'Brodovich $\mathrm{H}$, Coates $\mathrm{G}$, Marrin $\mathrm{M}$ : Effect of inspiratory resistance and PEEP on sup 99m Tc-DTPA clearance. J Appl Physiol 1986; 60:1461-1465 [Context Link]

29. Nieman G, Ritter-Hrncirik C, Grossman Z, et al: High alveolar surface tension increases clearance of technetium $99 \mathrm{~m}$ diethylenetriamine-pentaacetic acid. J Thorac Cardiovasc Surg 1990; 100:129-133 [Context Link]

30. Calderwood HW, Modell JH, Rogow L, et al: Morphologic and biochemical changes in dogs after ventilation with Caroxin-D fluorocarbon. Anesthesiology 1973; 39:488-495 Ovid Full Text | 360 Link| Bibliographic Links| [Context Link] 

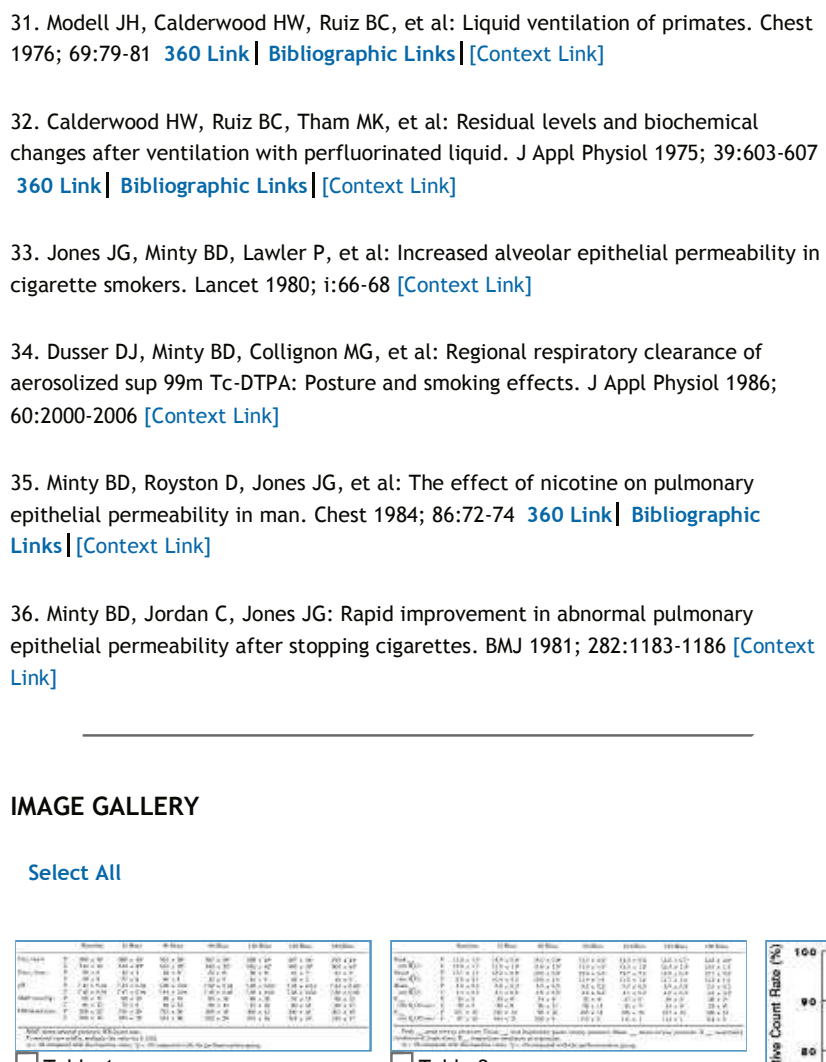

$\square$ Table 2

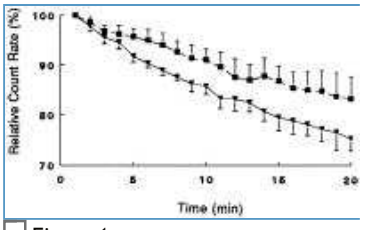

Back to Top

4 Previous Article | Table of Contents | Next Article 